\title{
THE RESERVOIR RUNOFF FORECAST WITH ARTIFICIAL NEURAL NETWORK BASED ON ANT COLONY OPTIMIZATION
}

\author{
WAN, F. ${ }^{1}$ - WANG, F. Q. $.^{*}-$ YUAN, W. L. ${ }^{2}$ \\ ${ }^{I}$ Department of Water Resources, North China University of Water Resources and Electric \\ Power, Zhengzhou 450045, China \\ ${ }^{2}$ School of Water Conservancy and Environment, Zhengzhou University \\ Zhengzhou 450001, China \\ *Corresponding author \\ e-mail:wangfuqiang@ncwu.edu.cn \\ (phone: +137-037-14661; fax:+371-693-10092) \\ (Received 30 ${ }^{\text {th }}$ Nov 2016; accepted $6^{\text {th }}$ Apr 2017)
}

\begin{abstract}
The results of reservoir water supply optimal dispatching depend to a great extent on the prediction of uncertain factors. Aiming at the problems of reservoir runoff forecast, an artificial neural network based on ant colony optimization is presented. The perturbation strategy is added in this new hybrid algorithm, the improvement ant colony optimization can combine the strong memory and associative ability of artificial neural network with positive feedback of ant colony optimization, and can overcome the shortcomings in the respect of lack of pheromone at initial stage and easiness of local optimization of ant colony optimization. It is applied to six reservoirs optimization water supply dispatching in the lower Luan he river. A practical example shows that this new hybrid algorithm is rational, reliable and high accuracy when it is used to solve the problem of reservoir runoff forecast, at the same time, the training precision is high. So, this new algorithm is applied to forecast the reservoir runoff. Keywords: back-propagation neural network; uncertain factors; water supply dispatching; perturbation strategy; Luan he river
\end{abstract}

\section{Introduction}

The forecast of reservoir flow forecasts the hydro-logical regime in the future, which is mainly based on the hydro-logical and meteorological data and adopts the methodology of physical analysis and statistics. The traditional forecasting models mainly include genetic analysis and hydro-logical statistics. In recent years, with the development of computer technology, the methods of hydro-logical forecasting (Wan et al., 2016; Wei et al., 2013; Chang et al., 2013; Ahmad et al., 2017) mainly include chaotic analysis, grey system prediction, extension classified prediction method, artificial neural network method, wavelet analysis and the mixture of these methods. In this paper, we use the strong memory of neural network, positive feedback of association ability and ant colony algorithm, at the same time, we get rid of such problems as lacking information pheromone in the initial stage, getting trapped in a local optimum easily of ant colony algorithm in solving the model. In ant colony algorithm, we increase perturbation strategy, combine neural network and ant colony algorithm organically to propose a neural network algorithm based on ant colony optimization, forecasting reservoir runoff. 


\section{Back-propagation neural network based on ant colony optimization}

BP algorithm (Mohammad et al., 2013; He et al., 2014; Maryam et al., 2016) has the following advantages in theory, simpler network construction, strong memory and association ability, more stable state (Li et al., 2008; Wang and Zhang, 2010). But in the practical application of hydro-logic forecasting, there are some problems. The input of the new sample has an impact on the studied samples. We rely on experience to select the weights of the initial value and the number of neurons (Yi et al., 2013; Yang et al., 2016). Ant Colony Optimization or ACO (Qun et al., 2013; Kwang et al., 2014; Kleinkauf et al., 2015; Zhao et al., 2016; Vijay et al., 2016), is a probabilistic search algorithm with the advantages such as being parallel, positive feedback, strong robustness and excellent distributed computer system and so on.

\section{Optimization model}

In view of the above problems, the BP neural network is improved as follows: combining weights and parameters (A-BP) by using ACO and BP network, the network is trained, the training samples were normalized.

\section{Basic principles of neural networks}

Artificial neural network or ANN (Chen and Chang, 2009; Zhao et al., 2009), a nonlinear information parallel processing system (He et al., 2014; Shafaei and Kisi, 2014; Gharibreza, 2017) for simulating the structure and function of the neural network of the human brain, is composed of a large number of processing units (artificial neural networks) connected to each other and into the network, which has the characteristics of self-learning, self-adaptation, self-organization and so on (Khan et al., 2017). The neural network model was applied to the concept of energy function present a method for judging the stability of network in 1982, at the same time, it puts forward the concept of hidden unit. the weight adjusted error Back-Propagation algorithm for mufti- layer feed forward network was proposed In 1986, so that the research of artificial neural network is further studied. This kind of feed-forward network based on BP algorithm, generally called BP network, is one of the most popular neural networks currently. It can be used to approach the complex nonlinear function with high precision, which has the advantages such as simpler network construction, strong memory and association ability, more stable state.

\section{The structure of BP neural network}

BP network is generally composed of an input layer, an output layer, one or more of the hidden layer. There are a number of nodes in each layer. Fig. 1 is the structure of BP network.

With an input layer of neurons, a hidden layer of neurons, an output layer of neurons. $x_{1}, x_{2}, \ldots, x_{n}$ are the input of the neural network, $y_{1}, y_{2}, \ldots, y_{1}$ are the output of the neural network? The information in the hidden layer can be used as input information in a non-linear way, and the output layer is transmitted to the output layer. The hidden layer is usually generated by the excitation function output information. Its excitation function is generally used Sigmund function. The information in the input layer must be transmitted to the hidden layer. The calculation process of BP network is composed of two parts, the forward calculation process and the reverse calculation 
process. Firstly, the input layer, hidden layer and output layer are calculated by layer by layer, then the output of the sample is get Secondly, the error between the expected sample output and the neural network calculation results is calculated by the error calculation formula. Finally, according to the error to adjust the network weight, the connection weights are corrected by the forward and backward, until the error meets the specified requirements.

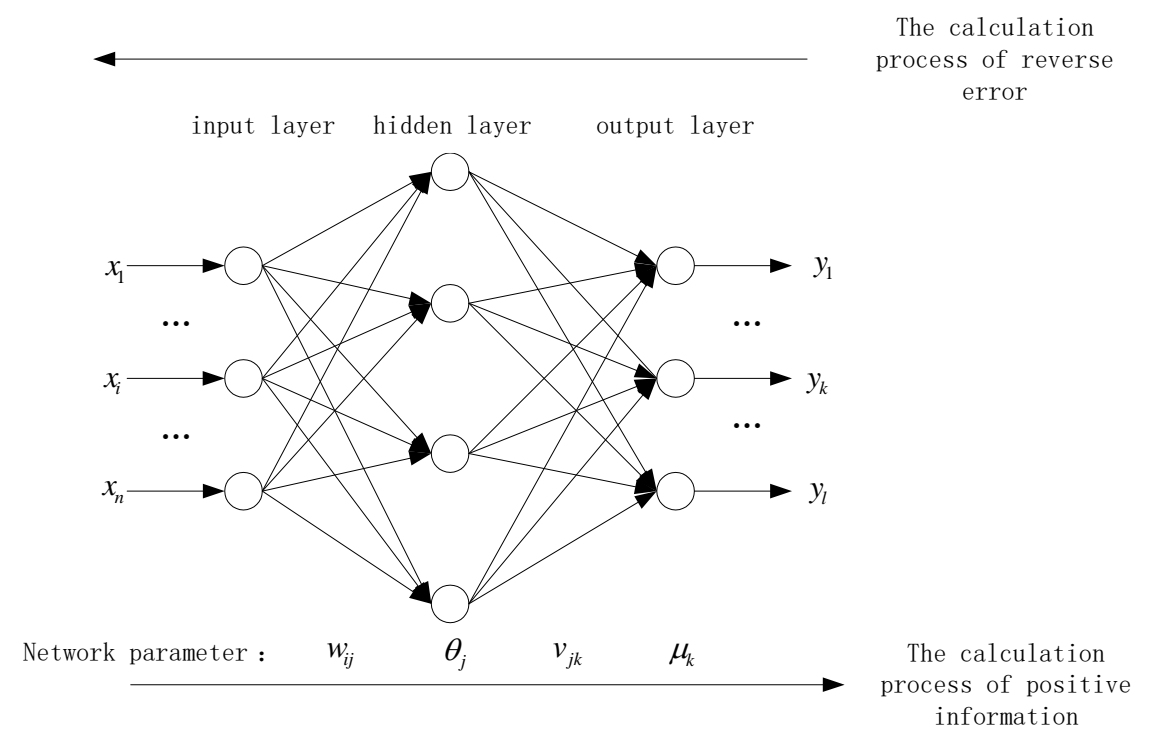

Figure 1. The structure of BP Neural network

(1) Random generation of initial threshold and weight

After determining the number of input layer neurons of BP neural network structure, the number of hidden layer neurons, the number of neurons in the output layer $(\mathrm{n}, \mathrm{m}, 1)$. According to the basic principle of neural network, the parameters of neural network include the weights $w_{i j}(i=1,2, \ldots, n ; j=1,2, \ldots, m)$ from the input layer $i$ to the hidden layer unit $j$; activation threshold $\theta_{j}(j=1,2, \ldots, m)$ of hidden layer units $j$; the connection weights $V_{j k}(j=1,2, \ldots, m ; k=1,2, \ldots, l)$ of the hidden layer units $j$ to the output layer units $k$; activation threshold $\mu_{k}(k=1,2, \ldots, l)$ of output layer cell $k$.The initial weights of the network are generally randomly generated between $[-1,1]$.

(2) Output of $Q$ training samples

Suppose there are training sample, the input $x_{i q}(i=1,2, \ldots, n)$ of the first sample $q(q=1,2, \ldots, Q)$ is transferred to the hidden layer. The output information $h_{j q}$ of the hidden layer $h_{j q}$ is obtained by the activation function $f_{1}(s)$.

$$
h_{j q}=f_{1}\left(s_{j q}\right)=f_{1}\left(\sum_{i=1}^{n} w_{i j} X_{i q}-\theta_{j}\right) \quad j=1,2, \ldots, m ; q=1,2, \ldots, Q
$$

Activation function generally selects Sigmoid function.

$$
f(s)=\frac{1}{1+e^{-x}}
$$


The output information $h_{j q}$ of the hidden layer $h_{j q}$ is transferred to the output layer, and the network output $O_{k q}$ is obtained.

$$
O_{k q}=f_{2}\left(r_{k q}\right)=f_{2}\left(\sum_{j=1}^{m} V_{j k} h_{j q}-\mu_{k}\right) \quad k=1,2, \ldots, 1 ; q=1,2, \ldots, Q \text { (Eq.3) }
$$

(3) Error calculation

There are usually some errors between the expected value $y_{k q}$ and the output $\mathrm{O}_{\mathrm{kq}}$ of the neural network. The error function $E(q)$ of the sample $Q$ can be expressed as:

$$
E_{q}=\frac{1}{2 q} \sum_{q=1}^{Q} \sum_{k=1}^{l}\left(O_{k q}-y_{k q}\right)^{2} \quad k=1,2, \ldots, l ; q=1,2, \ldots, Q
$$

If the error meets certain accuracy requirements, the network learning ends, otherwise the network weights and thresholds are adjusted according to the error.

(4) To adjust the weights and threshold

The number of neurons in the neural network has been determined; it can reduce the error through the adjustment of threshold value and weight to have achieved the goal of improving the accuracy of calculation. $W$ can be modified, Because the error function $E_{q}$ changes along with the negative gradient $W$, so the correction value is $\Delta W$.

$$
\Delta w(t+1)=w(t+1)-w(t)=-\eta \frac{\partial E_{q}}{\partial w}
$$

In the formula, $\eta$ is the learning rate, the range is from 0 to $1 . W$ is certain weight or threshold.

Under normal circumstances, if $\eta$ becomes larger, although the convergence of the network is fast. There will be oscillations; if $\eta$ becomes smaller, the convergence of the network becomes slower. In order to avoid this situation, we can by inertia factor $\alpha$, its values range from 0 to 1 , which is analysed by (5).

$$
\Delta w(t+1)=-\eta \frac{\partial E_{q}}{\partial w}+\alpha \Delta w(t)
$$

For hidden layer:

$$
\frac{\partial E_{q}}{\partial \theta_{k}}=\delta_{j q}^{\prime}=f_{1}^{\prime}\left(s_{j q}\right) \cdot \sum_{k=1}^{l} \delta_{k q} v_{j k}, \quad \frac{\partial E_{q}}{\partial w_{i j}}=-\delta_{j q}^{\prime} x_{i q}
$$

Therefore, from the input layer to the hidden layer weights, the hidden layer threshold of the modified formula is: 


$$
\begin{gathered}
\Delta w_{i j}(t+1)=\eta \delta_{j q}^{\prime} x_{i q}+\alpha \Delta w_{i j}(t) \\
\Delta \theta_{j}(t+1)=-\eta \delta_{j q}^{\prime}+\alpha \Delta \theta_{j}(t)
\end{gathered}
$$

For output layer neurons:

$$
\frac{\partial E_{q}}{\partial \mu_{k}}=\delta_{k q}=\left(y_{k q}-O_{k q}\right) \cdot f_{2}^{\prime}\left(r_{k q}\right), \quad \frac{\partial E_{q}}{\partial v_{j k}}=-\delta_{k q} h_{j q}
$$

Therefore, the correction formula from the hidden layer to the weight of the output layer and the threshold of output layer is:

$$
\begin{gathered}
\Delta v_{j k}(t+1)=\eta \delta_{k q} h_{j q}+\alpha \Delta v_{j k}(t) \\
\Delta \mu_{k}(t+1)=-\eta \delta_{k q}+\alpha \Delta \mu_{k}(t)
\end{gathered}
$$

After adjusting the weights, if the error does not meet the accuracy requirements, the procedure (2) is transferred to calculation, until the error meets the specified accuracy requirements.

\section{The basic principle of ant colony optimization algorithm}

Ant Colony Optimization or ACO, an optimization algorithm (Blum and Dorigo, 2004; Du et al., 2014) based on ant foraging principle, has the advantages (Deng and Peng, 2010; Yuan and Qu, 2013) such as parallel, positive and negative feedback, strong robustness and excellent distributed computer system. In initial time, the pheromone equals on all paths, the pheromone trail intensity $\tau_{i j}(0)=C_{0}\left(C_{0}\right.$ is a constant $)$ from the nest $i$ to the food source $j$. In the course of the movement, the ant $k(k=1,2, \ldots, m)$ decides the direction of the transfer according to the amount of information on each path. At the moment of $t$, the transition probability $P_{i j}^{k}(t)$ of the ant $k$ in the path $i$ and the path $j$ is:

$$
P_{i j}^{k}(t)=\left\{\begin{array}{c}
\frac{\tau_{i j}^{\alpha}(t) \eta_{i j}^{\beta}(t)}{\sum_{\text {seallowed }_{k}} \tau_{i s}^{\alpha}(t) \eta_{i s}^{\beta}(t)}, j \in \text { allowed }_{k} \\
0, \text { otherwise }
\end{array}\right.
$$

In the formula, allowed ${ }_{k}=\{0,1, \ldots, n-1\}$ is the next step to allow the ant $k$ to choose the target; $\tau_{i j}$ is pheromone trail strength of edges $(i, j) ; \eta_{i j}$ is heuristic factor for edge $(i, j), P_{i j}^{k}$ is transition probability for the ant $k, \alpha, \beta$ are two parameters. The relative importance of the information and heuristic information accumulated during the process of the ants in the selection of ants reflected respectively. The amount of pheromone on each path is adjusted according to the following formula.

$$
\tau_{i j}(t+1)=(1-\rho) \tau_{i j}(t)+\rho \Delta \tau_{i j}(t, t+1)
$$




$$
\Delta \tau_{i j}(t, t+1)=\sum_{k=1}^{m} \Delta \tau_{i j}^{k}(t, t+1)
$$

In the formula, $\Delta \tau_{i j}^{k}(t, t+1)$ is the pheromone of the ant $k$ to stay on the path $(i, j)$ at all times of $(t, t+1)$. The value depends on the merits and virtues of the ants. The shorter the path is, the more pheromone releases. $\Delta \tau_{i j}(t, t+1)$ is increment of the pheromone amount of the loop path $(i, j) . \rho$ is attenuation coefficient of pheromone. Usually, we set $\rho<1$ to avoid an unlimited increase in the amount of path on the path.

In this paper, we will use the random perturbation strategy to prevent the stagnation of ant colony algorithm. The random selection probability needs to adjust dynamically. Approximate optimal solutions are obtained. The calculation time is shortened and the calculation efficiency is improved.

The ants' choices of their paths are random. Generally, they choose the path of transition probability, but the optimal path is not always selected, which results in a subsequent search for stagnation. Because the current optimal path information is more than the fact that doesn't find the optimal path pheromone number, with the increase of the number of iterations, the actual optimal path is less and less, so the probability of choosing this path becomes smaller and smaller. Considering the stagnation of the algorithm as well as based on the characteristics of the above ants in the choice of path at the same time, "disturbance factor" will be added to interfere with the ant selection path. The pheromone is not the most path to a certain probability of random selected, this path may be the best. At the same time, the probability of random selection in evolutionary computation which needs to be adjusted dynamically to increase the diversity of the selected paths, but the probability of the maximum path of the pheromone is calculated separately to prevent leakage of the optimal paths. The transition probability of the perturbation strategy can be formulated as follows:

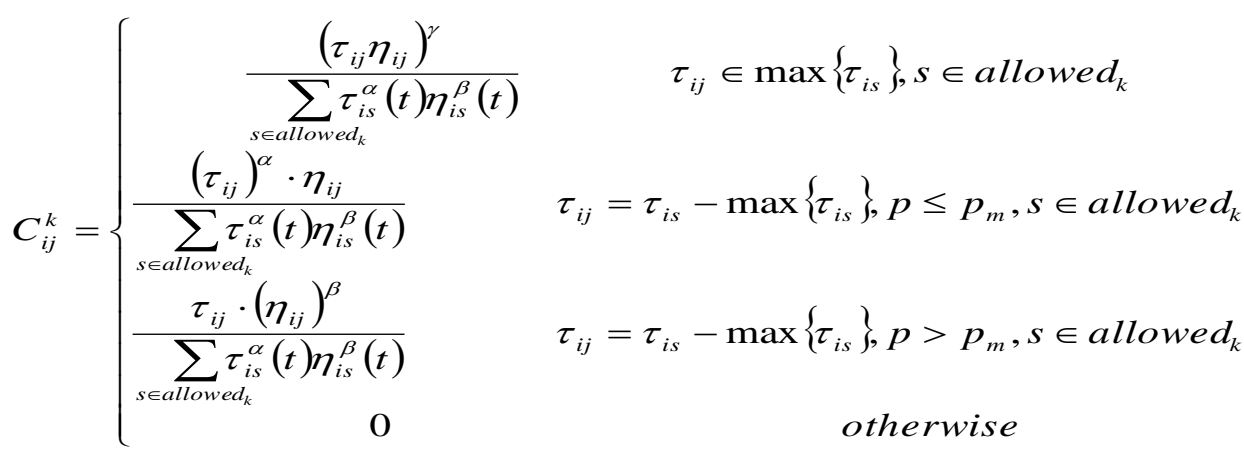

In the formula, $\gamma$ is a reverse exponential perturbation factor; $p_{m} \in(0,1)$ is random mutation rate; $p$ abides to the uniform distribution of random variables. The formula indicates that ants in an iterative process can choose a number of paths. The formula (Eq.13) is used to calculate the transition probability on the path of the pheromone. In a random choice, we can calculate the transition probability on the alternative paths, the perturbation strategy is a combination of random selection and deterministic selection. The random selection makes the choice of the path and the calculation of the strong randomness, deterministic choice refers to the path that the ant will choose the most transition probability. 


\section{Methods solution}

\section{The training samples were normalized}

Because the input of the physical quantity is not the same, so the difference is very big, so the input data should be normalized before calculating to convert it from 0 to 1 (logarithmic S curve). Normalized change is according to the following formula:

$$
T=T_{\min }+\frac{T_{\text {max }}-T_{\min }}{X_{\text {max }}-X_{\min }}\left(X-X_{\min }\right)
$$

In the formula, $X$ is original input data. $X_{\max }, X_{\min }$ are the maximum and minimum values respectively. $T$ is transformed data, $T_{\max }, T_{\min }$ are the maximum and minimum values settled respectively. $T_{\max }$ usually takes the rang from 0.8 to $0.9, T_{\min }$ is $1-T_{\max }$.

\section{Ant colony algorithm (ACO) to optimize the BP network}

Firstly, ant colony algorithm is used to optimize the initial weights and network structure of BP neural network. The hydrological forecasting model is established. The network training and fitting experimental data should be gotten. Then the algorithm is applied to train the network samples so that the network output error is minimized. The effective improvement of BP neural network can easily fall into the local minimum value and the convergence speed is slow and all so defects. Suppose there are $m$ parameters to be optimized of BP neural network, the parameters are arranged in order: $p_{1}, p_{2}, \ldots \ldots p_{m}$. According to any parameter, initialization $\mathrm{N}$ any nonzero value, the set $I_{p i}$ is constituted. Suppose the ant number is $S$, all ants search for food by randomly selected element from first set, then return to the nest after finding food. Repeat this step, until all ants collect the same route, the optimum solution is obtained of this network. The specific calculation steps are as follows:

Step1: The BP neural network model is established, including the number of network layers, the number of nodes, the range and the sample size to be optimized.

Step2: Initialize ant colony. The parameters are uniformly dispersed. Initialization the path that according to discretize data. Then the complete path is established. The Pheromone trail intensity is $\tau_{i j}(0)=C_{0}\left(C_{0} i s\right.$ constant $)$. The combination of discrete points of each parameter is defined as the path of ants that is a solution to the problem.

Step3: Cyclic iteration of ant colony algorithm. At the end of each iteration, generate random numbers $q$ of the range from 0 to 1 , make a comparison of explored relative importance threshold value $q_{0}\left(0 \leq q_{0} \leq 1\right)$ of the new path with the prior knowledge. If $q \leq q_{0}$, the parameters of each weight are randomly variant according to the formula (Eq.16), random mutation is for new weight discrete points to get added to the collection S. If $q \geq q_{0}$, select the weight according to the formula (Eq.13).

Step4: All ants set out from set $I_{p i}$, follow the path rule to find element in order, finally the food source is found. The rule of route choice is: All ants $k(1,2, \ldots \ldots M)$ arbitrary choose $j^{\text {th }}$ with certain probability. The probability formula:

$$
\operatorname{Pr} o b\left(t_{j}^{k}\left(I_{p i}\right)\right)=\left(t_{j}\left(I_{p i}\right)\right) / \sum_{u=1}^{N} t_{u}\left(I_{p i}\right)
$$


Step5: When all the ants are completed, we input training sample. According to the formula (Eq.14) (Eq.15), the weight parameters were updated.

Step6: A set of the best weights that find by the ant colony algorithm are used as the initial weights of the BP algorithm. The error between the network output and the actual output is calculated. And the error is propagated from the output layer to the input layer, adjust weights, if the error reaches a predetermined precision or satisfy the maximum iterations number $T$, the algorithm is over. Otherwise, re-select ant colony to Step2.

The flowchart of an artificial neural network based on ant colony optimization is described in Figure 2.

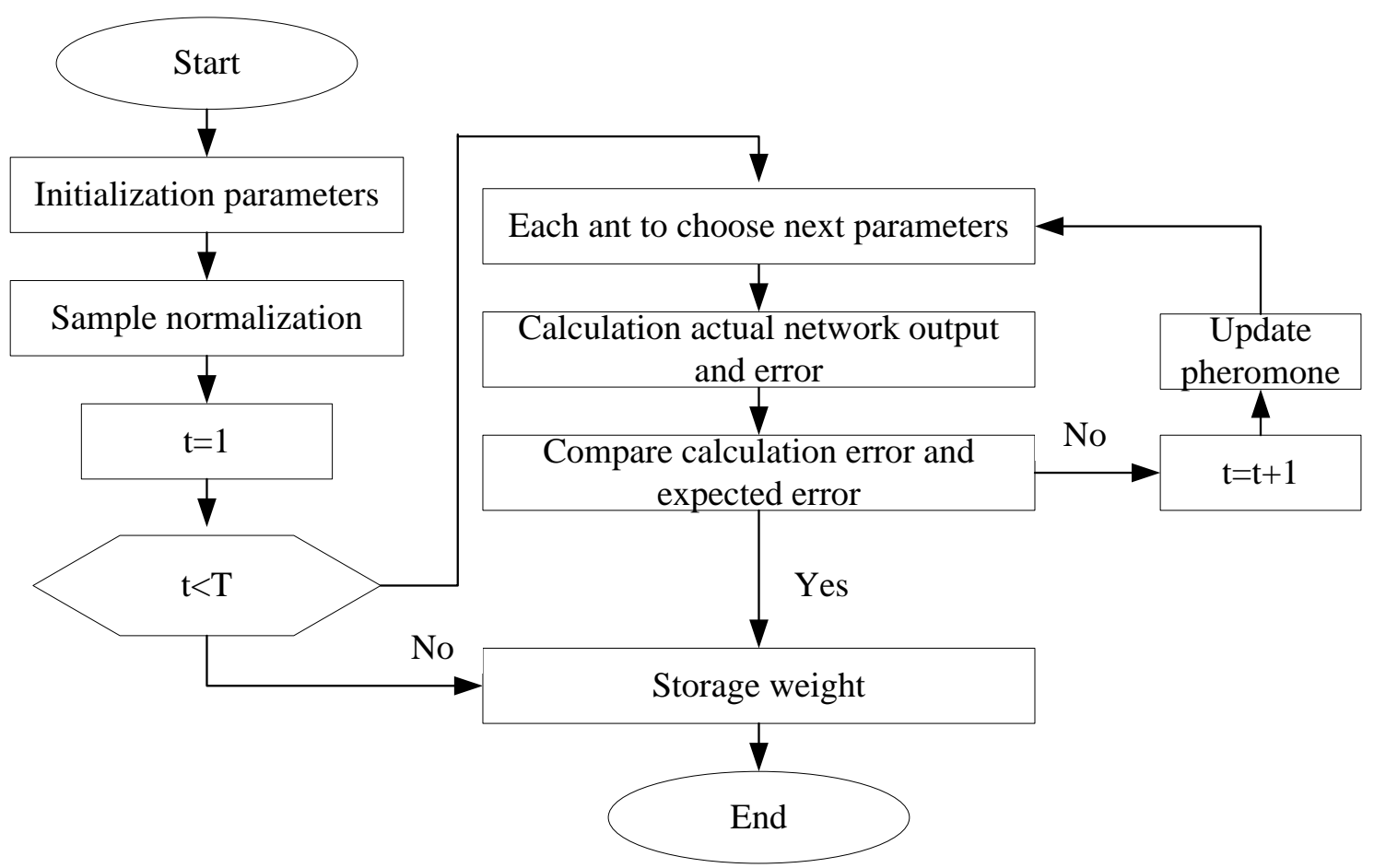

Figure 2. The flowchart of an artificial neural network based on ant colony optimization

\section{Case study}

The LuanHe River is rich in water resources in Northern China. The total precipitation ranges between $129 \times 10^{8} \mathrm{~m}^{3}$ and $12.7 \times 10^{8} \mathrm{~m}^{3}$, and has uneven distribution of inter-annual variations, with characteristic of a typical semi-arid and semi-humid region. The lower LuanHe River is the most important source that supply water to TianJin and TangShan which face severe challenge of the imbalance between supply and demand of water resource the situation is aggravated day by day. Five reservoirs have been constructed and are delivering an annual flow of 2873 million cubic meter water into the cities. The annual water deficiencies are 1025 million cubic meters. The flow supplies water for agricultural, industrial and municipal uses.

Each reservoir's characteristics are as follows:

PanJiaKou reservoir is a carryover storage reservoir with total storage capacity is $29.3 \times 10^{8} \mathrm{~m}^{3}$, and its regulating the function is perfect; DaHeiTing reservoir is located in the PanJiaKou reservoir downstream, which is a annual regulation reservoir, and the 
total storage capacity is $3.37 \times 10^{8} \mathrm{~m}^{3}$; YuQiao reservoir is a larger-scale reservoir in TianJin, and total storage capacity is $15.59 \times 10^{8} \mathrm{~m}^{3}$; QiuZhuang reservoir which is an intermediate regulation reservoir and Taolinkou reservoir belong to QinHuangDao. To augment the water supply in the basin and to keep up with the increasing demand, inter-basin transfers have been implemented, by bring them into full play in a multiply connected reservoirs. The main project characteristic indexes of each water supply reservoir are described in Table 1.

Table 1. Main project characteristic indexes of each water supply reservoir (Unit: $10^{8} \mathrm{~m}^{3}$ )

\begin{tabular}{|c|c|c|c|c|c|}
\hline $\operatorname{Res}$ & oirs & $\begin{array}{l}\text { Dead } \\
\text { storage }\end{array}$ & $\begin{array}{l}\text { Active } \\
\text { storage }\end{array}$ & $\begin{array}{c}\text { Total } \\
\text { storage }\end{array}$ & Water supply task \\
\hline \multirow{2}{*}{$\begin{array}{l}\text { Donor } \\
\text { reservoirs }\end{array}$} & PanJiaKou & 3.31 & 19.50 & 29.30 & \multirow{2}{*}{$\begin{array}{c}\text { No direct user, supply water to recipient } \\
\text { reservoirs }\end{array}$} \\
\hline & DaHeiTing & 1.13 & 2.07 & 4.73 & \\
\hline \multirow{3}{*}{$\begin{array}{l}\text { Recipient } \\
\text { reservoirs }\end{array}$} & YuQiao & 0.36 & 3.85 & 15.59 & \multirow{3}{*}{$\begin{array}{l}\text { Supply water to Tianjin of industry and } \\
\text { domestic life } \\
\text { Supply water to Tangshan of industry and } \\
\text { domestic life } \\
\text { Supply water to Qinhuangdao of industry } \\
\text { domestic life and agriculture of lower } \\
\text { LuanHe river }\end{array}$} \\
\hline & QiuZhuang & 0.008 & 0.65 & 2.04 & \\
\hline & Taolinkou & 0.51 & 7.09 & 8.59 & \\
\hline
\end{tabular}

\section{Results and discussion}

Neural network model based on ant colony optimization makes a prediction of the runoff in the lower reaches of the Lutheran River Reservoir. Select Panjiakou Reservoir, Daheiting and Taolinkou Reservoir respectively (Given the limited space available, prediction of bridge reservoir, the results of Qiuzhuang reservoir and Yuqiao reservoir are not listed.) The research object is the data of inflow runoff in the 26 year. January in 1975 to December in 1995 as a training sample and regard data for network fitting test of 5 years from January in 1996 to December in 2000 as network fitting test (Ghosh and Dash, 2017).

Design guidelines for ACO-BP neural networks: The data is first trained by ACO algorithm, after training is completed, then the neural network is trained by the BP algorithm, the learning rate of BP algorithm is as small as possible so as to perform fine search behavior, the number of training of BP algorithm as much as possible not to exceed the average absolute error, the maximum relative error and mean square difference of 1000 times, because most of the global search work has been completed by the ACO algorithm, the $\mathrm{BP}$ algorithm only needs to perform a more detailed local search of the search's finishing work. BP neural network training

Parameters set as follows: the maximum number of training to take 800 , learning efficiency to take 0.1 , learning output error target to take 0.001 . The ant colony algorithm is initialized as follows: the number of ants is 50, the important factor of pheromone is 1 , the importance factor of the heuristic function is 5 , the pheromone factor is 0.1, and the maximum number of iterations is 200, as shown in Figs. 3-8. 


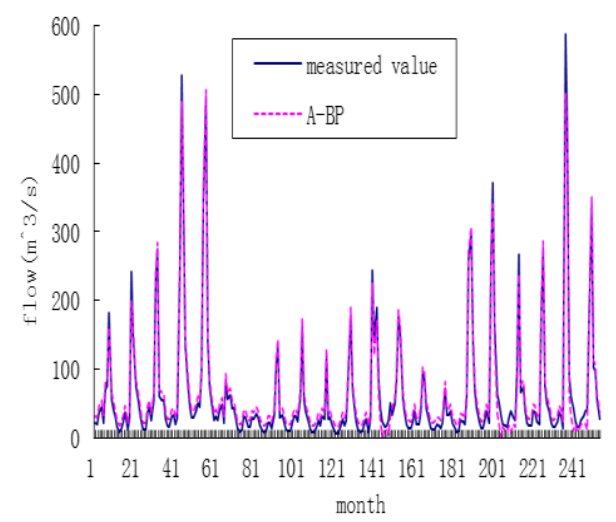

Figure 3. The comparison figure between measured and trained values of monthly inflow of PanJiaKou (January 1975 - December 1995)

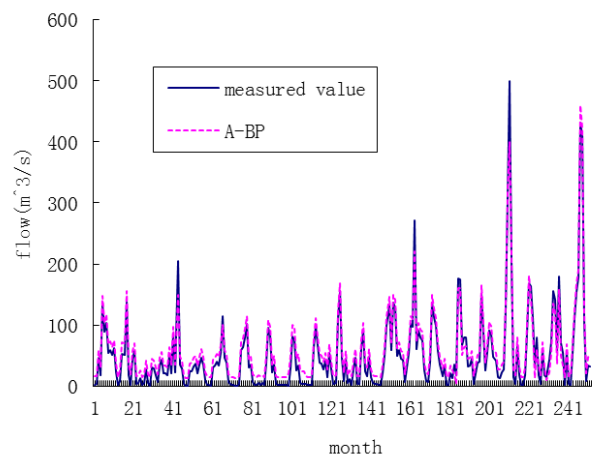

Figure 5. The comparison figure between measured and trained values of monthly inflow of DaHeiTing (January 1975 -

December 1995)

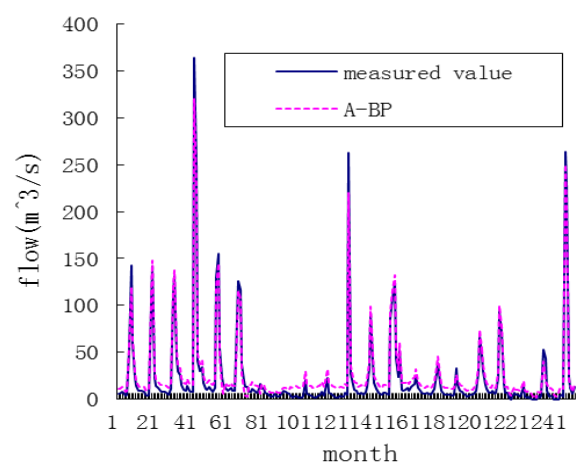

Figure 7. The comparison figure between measured and trained values of monthly inflow of Taolinkou of Taolinkou reservoir(January 1996-2000)

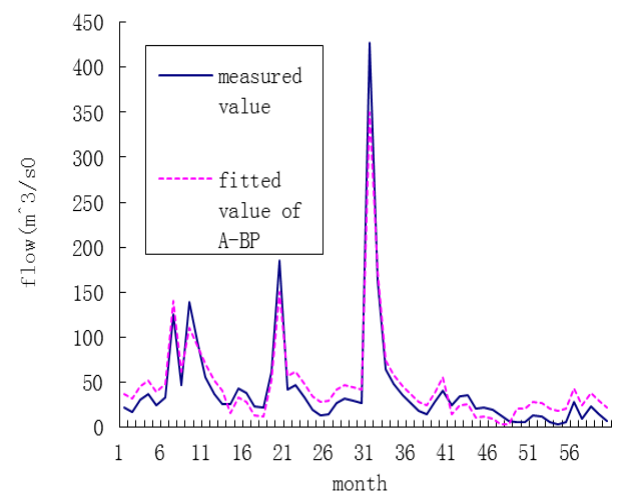

Figure 4. The comparison figure between measured the fitted values of monthly inflow reservoir of PanJiaKou reservoir (January $1996-2000)$

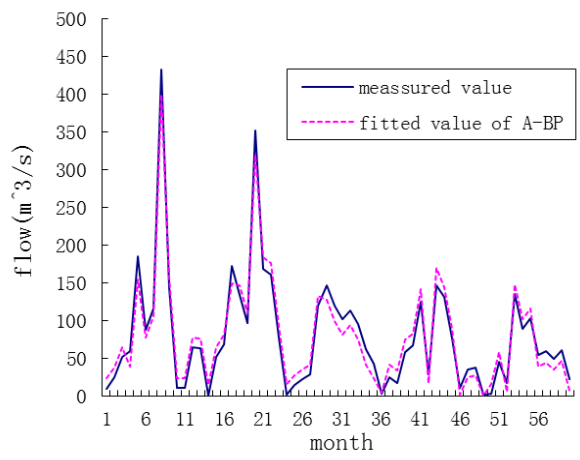

Figure 6. The comparison figure between measured the fitted values of monthly inflow reservoir of DaHeiTing reservoir(January 1996-2000)

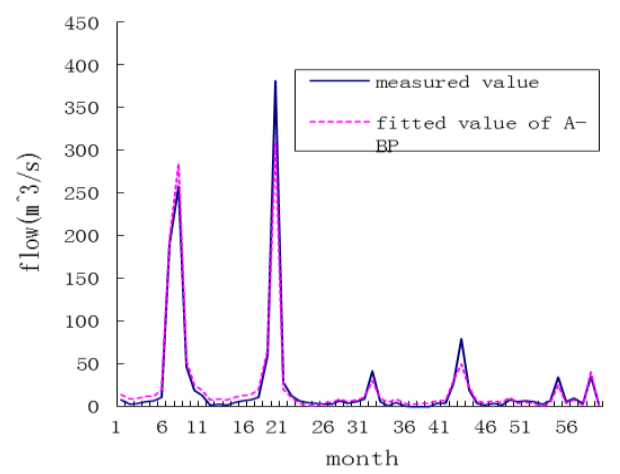

Figure 8. The comparison figure between measured the fitted values of monthly inflow reservoir(January 1975 - December 1995)

The Panjiakou Reservoir from the year 2001 to 2005 of annual runoff were predicted and compared with the actual value. The forecast effect is shown in Fig. 8. 


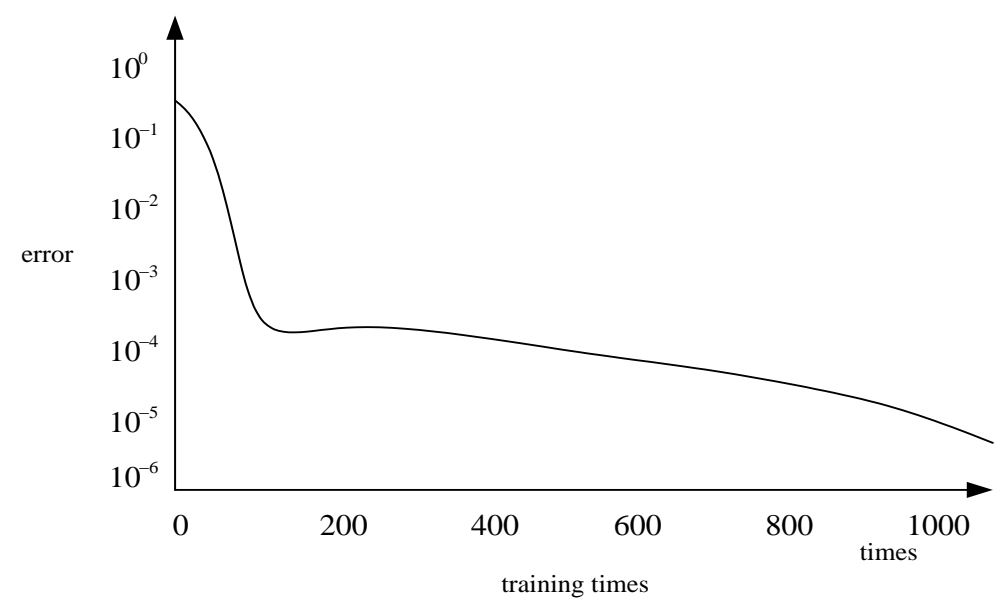

Figure 8. The figure of error changing about network model trained values

The analysis of Figure 8 shows, the BP neural network model based on ant colony optimization is reasonable and reliable.it can make a prediction of reservoir runoff; According to the result of calculation Formula (4), the error between the fitting results and the measured values is compared within $10 \%$. It shows that the training accuracy is higher and can meet the demand, so the runoff of the reservoir can be predicted.

In order to explain the prediction effect of different algorithms, the average absolute error (AAE), maximum relative error(MRE) and root mean square (RMS) are proposed.

$$
\begin{aligned}
& A A E=\frac{1}{n} \sum_{k=1}^{n}\left|x_{k}-x^{\prime}\right| \\
& M R E=\max \left|x_{k}-x_{k}^{\prime}\right| \\
& R M S=\sqrt{\frac{\sum_{k=1}^{n}\left(x_{k}-x_{k}^{\prime}\right)^{2}}{n}}
\end{aligned}
$$

In the formula, $x_{k}$ is the network output value; $x_{k}^{\prime}$ is the true value; $n$ is the total number of sample. The error comparison of several algorithms is described in Table 2 with Panjiakou reservoir.

Table 2. The error comparison of several algorithms with Panjiakou reservoir

\begin{tabular}{cccc}
\hline $\begin{array}{c}\text { Difference } \\
\text { algorithms }\end{array}$ & $A A E$ & $M R E$ & $R M S$ \\
\hline BP & 6.24 & 9.62 & 7.63 \\
PSO-BP & 5.38 & 8.35 & 6.32 \\
ACO-BP & 4.25 & 7.01 & 5.24 \\
\hline
\end{tabular}


Through the comparison of error indicators which include average absolute error, maximum relative error and root mean square difference, etc, we can see from Table 2, the optimized BP neural network algorithm based on ant colony algorithm proposed in this paper has better fit performance and predictability, mainly because the PSO algorithm in the context of the search cannot easily find the local optimization, and easy to fall into them, so the accuracy of its operation is not very satisfactory. In the search process, it will find the optimal value, but there is no guarantee that this is the global optimal, the ant colony algorithm is relatively good in this respect. Thus, the optimized BP neural network algorithm based on ant colony algorithm improves the accuracy of hydrological prediction to reduce the error.

\section{Conclusions}

In this paper, the ant colony optimization algorithm is introduced into the neural network. Random disturbance strategies are added. In this paper, we make full use of the strong memory and associative ability of neural network as well as the characteristics of state stability and the local search ability of ant colony algorithm. At the same time, we get rid of such problems as lacking information pheromone in the initial stage, getting trapped in a local optimum easily of ant colony algorithm in solving the model, improving the accuracy of calculation. The algorithm is applied to the prediction of runoff in reservoir. The error between the fitting results and the measured values is compared to get the result within $10 \%$, which is able to meet the requirements. In this paper, the neural network model based on ant colony optimization is reasonable and reliable, which can predict reservoir runoff, training accuracy is higher, so we can predict the runoff of the reservoir. In spite of this, the forecast of uncertain factors such as reservoir runoff are uncertain, but in the actual operation, water and water has great randomness, so we need further stud the real-time forecasting of reservoir runoff.

Acknowledgements. This study was supported by the National Key Research and Development Program of China (2016YFC0401401), Major Research Plan of the National Natural Science Foundation of China (91547209), the National Natural Science Foundation of People's Republic of China (51509089, 51379078,

51409103, 51579101), the Science-tech Innovation Talents in University of Henan Province (15HASTIT044).

\section{REFERENCES}

[1] Ahmad, N., Hussain T., Awan A.N., Sattar A., Arslan, C., Tusief M.Q., Mariam Z. (2017): Efficient and Eco-friendly Management of biodegradable Municipal Solid Waste (MSW) using naturally aerated Windrow Composting Technique in District Lahore Pakistan. Earth Science Pakistan. 1(1): 01-04.

[2] Blum, C., Dorigo, M. (2004): Deception in ant colony optimization. In: Ant colony optimization and swarm intelligence. - Springer Berlin/Heidelberg 3172: 118-129.

[3] Chang, J. X., Bai, T., Huang, Q., Yang, D. W. (2013): Optimization of water resources utilization by by PSO-GA. - Water Resource Manage (10): 3525-3540.

[4] Chen, Y. H., Chang, F. J. (2009): Evolutionary artificial neural networks for hydrological systems forecasting. - Journal of hydrologic 367:125-137.

[5] Deng, X. Y., Peng, Y. (2010): Review on application of particle swarm optimization to operation of reservoirs. - Advances in Science and Technology of Water Resources 30(5): 90-94. 
[6] Du, P. Z., Tang, Z.M., Sun, Y. (2014): An object-oriented multi-role ant colony optimization algorithm for solving TSP problem. - Control and Decision 29(10): 1729-1736.

[7] Gharibreza M. (2017): Late Holocene evolution of the HendijanDelta (north of the Persian Gulf). Geology, Ecology, and Landscapes. 1(2), 71-76.

[8] Ghosh S., Das A. P. (2017): Bioleaching of manganese from mining wasteresidues using Acinetobacter sp., Geology, Ecology, and Landscapes. 1(2), 77-83.

[9] He Z. F., Zhang Y. N., Guo Q. C., Zhao X. R.(2014): Comparative Study of Artificial Neural Networks and Wavelet Artificial Neural Networks for Groundwater Depth Data Forecasting with Various Curve Fractal Dimensions. Water Resources Management. 28(15): 5297-5317.

[10] He, Z. F., Zhang, Y. N., Guo, Q. C., Zhao, X. R. (2014): Comparative Study of Artificial Neural Networks and Wavelet Artificial Neural Networks for Groundwater Depth Data Forecasting with Various Curve Fractal Dimensions. - Water Resources Management. 28(15): 5297-5317.

[11] Khan A., Rehman, R., Rashid H., Nasir A. (2017): Exploration of Environmental Friendly adsorbents for Treatment of Azo Dyes from Textile Wastewater and its dosage optimization. Earth Science Pakistan. 1(1): 05-07.

[12] Kleinkauf, R., Houwaart, T., Backofen, R., Mann, M. (2015): antaRNA - Multi- objective inverse folding of pseudoknot RNA using ant-colony ptimization. - BMC Bioinformatics 16:389. DOI: 10.1186/s12859-015-0815-6

[13] Kwang S. Y., Seog Y. H.(2014): Modified Ant Colony Optimization for Topology Optimization of Geometrically Nonlinear Structures. International Journal of Precision Engineering and Manufacturing. 15(4): 679-687.

[14] Li, Y. B., Huang, Q., Xu, J. X., Zuo, W. B. (2008): Research on prediction of streamflow based on C-SVM. - Journal of Hydroelectric Engineering. 27(6): 42-47.

[15] Maryam S., Ozgur K.(2016): Predicting river daily flow using wavelet-artificial neural networks based on regression analyses in comparison with artificial neural networks and support vector machine models. Neural Computing and Applications. doi:10.1007/s00521-016-2293-9. 1-14.

[16] Mohammad A. A., Seyed R. S., Arash G.(2013): Retracted article: Combining artificial neural network and unified particle swarm optimization for oil flow rate prediction: case study. Neural Computing and Applications. 23(2): 565-565.

[17] Qun, Y., Lei, F., Xi, D. (2013): A randomly matched parallel ant colony optimization. - World Wide Web. 19(6): 1009-1022.

[18] Shafaei, M., Kisi, O. (2014): Predicting river daily flow using wavelet-artificial neural networks based on regression analyses in comparison with artificial neural networks and support vector machine models. - Neural Computing and Applications. DOI: 10.1007/s00521-016-2293-9

[19] Vijay R. A., Ravichandran C. S. (2016): Enhanced ant colony optimization to solve the optimal power flow with ecological emission. International Journal of System Assurance Engineering and Management. DOI: 10.1007/s13198-016-0471-x. 1-8.

[20] Wan, F., Yuan, W. L., Zhou, J. (2016): Derivation of Tri-level programming model for multi-reservoir optimal operation in inter-basin transfer-diversion-supply project. - Water Resour Manage.

[21] Wang, Y. M., Zhang, J. (2010): Run-off prediction model based on the chaotic and BP network. - Journal of Northwest A and F University 38(6): 200-204.

[22] Wei M. H., Wan F., Yang X., (2013). Study on the real-time multi-storage system water supply optimization dispatching. Yellow River. 35(8): 56-59.

[23] Yang, W. G., Hua, Y. L., Gao, Y. H. (2016): The pseudo-inverse matrix and its application in the improved BP neural network. - Fuzzy systems and mathematics 30(2): 184-190.

[24] Yi, Q. L., Zeng, H. N., Huang, H. F. (2013): Reservoir landslide deformation forecast using BP neural network. - Hydrogeology and Engineering Geology 40(1): 124-128. 
[25] Yuan, W. L., Qu, X. N. (2013): Application study on chaos ant colony optimization for operation of cascade reservoir power generation. - Journal of Hydroelectric Engineering 32(3): 47-54.

[26] Zhao, Z. P., Hou, M. T., Zhang, N. N. Gao, M. (2016): Multipath Routing Algorithm Based on Ant Colony Optimization and Energy Awareness. Wireless Personal Communications. DOI: 10.1007/s11277-016-3758-y. PP1-12.

[27] Zhao, Z. Y., Chow, T. L., Herb, W. R. (2009): Predict soil texture distributions using an artificial neural network model. - Comput Electron Agr. 65: 36-48. 